\title{
Endoscopic Myotomy: Advancements in Foregut Endoscopy
}

\author{
Marc F Catalano ${ }^{1 *}$, Sajjadh MJ Ali ${ }^{1}$, Todd D Wilson ${ }^{2}$, Hani Zamil ${ }^{1}$ and Brooks D Cash ${ }^{1}$ \\ ${ }^{1}$ Division of Gastroenterology, Hepatology and Nutrition, University of Texas, Houston, USA \\ ${ }^{2}$ Division of Minimally Invasive and Elective General Surgery, University of Texas, USA
}

*Corresponding author: : Marc F Catalano, Division of Gastroenterology, Hepatology and Nutrition, McGovern Medical School, 6431 Fannin Street, MSB 4.234, Houston, Texas, USA.

To Cite This Article: Marc F Catalano. Endoscopic Myotomy: Advancements in Foregut Endoscopy. Am J Biomed Sci \& Res. 2019 - 6(2). AJBSR. MS.ID.001003. DOI: 10.34297/AJBSR.2019.06.001003.

Received: 眥 October 09, 2019; Published: 制 November 08, 2019

\begin{abstract}
A number of diseases of the foregut are now being managed endoscopically instead of the traditional surgical methods. This brief review of literature deals with the advancements in endoscopy with reference to endoscopic myotomy.

Keywords: Endoscopy; Myotomy; POEM

Abbreviations: POEM: Per Oral Endoscopic Myotomy; LHM: Laparoscopic Heller Myotomy; EGJOO: Esophagogastric Junction Outflow Obstruction; SED: Spastic Esophageal Disorders; LES: Lower Esophageal Sphincter; LHM: Laparoscopic Heller Myotomy; SED: Spastic Esophageal Disorders; EGJ00: Esophagogastric Junction Outflow Obstruction; IRP: Integrated Relaxation Pressure; Endo FLIP: Endoluminal Functional Lumen Imaging Probe; GES: Gastric Electric Stimulation; PS: Pyloric Surgeries; G-POEM: Gastric Per Oral Endoscopic Myotomy; GCSI: Gastroparesis Cardinal Symptom Index
\end{abstract}

\section{Introduction}

The emerging field of endoscopic surgery can now be used to treat many diseases of the foregut which were earlier treated surgically. It is less invasive thereby having the advantages of decreased pain, length of hospital stays and earlier recovery as compared to the traditional surgical procedures. Recent advances like per oral endoscopic myotomy (POEM) have made long term outcomes comparable, if not better, as compared to surgical procedures in the treatment of achalasia [1]. This article will review the recent advances in foregut endoscopy especially with reference to endoscopic myotomy.

\section{Per oral endoscopic Myotomy (POEM)}

Esophageal motor disorders, in particular achalasia, have traditionally employed surgical myotomy (Heller). The endoscopic approach, called POEM, is now increasingly being used in achalasia. POEM involves making an incision in the mucosa after raising a submucosal bleb with saline and indigo carmine or methylene blue solution (Figure 1). A two-centimeter longitudinal incision is made on the mucosa to expose the submucosa using a through-the- scope knife which allows for simultaneous cutting, coagulation and injection. Once the submucosa is exposed, further dissection and injection allows for creation of a tunnel. This is generally started $10 \mathrm{~cm}$ above the lower esophageal sphincter (LES) and continued $2 \mathrm{~cm}$ beyond the LES. Once the tunnel is completed, selective cutting of the inner circular muscle is performed. Bleeding is often encountered and controlled using coagulation current. Closure of the mucosal incision can be done using endoluminal suturing devices (eg. Apollo Overstitch) or clips.

Achalasia: Standard POEM technique is suited for type I and type II achalasia. POEM can also deliver a longer incision compared to myotomy and hence is the preferred procedure for Achalasia type III. A meta-analysis of 22 studies comparing POEM and laparoscopic Heller myotomy (LHM) showed that POEM was more likely to be successful for type I and type III while the likelihood of success of POEM was similar to LHM for type II [2]. Another study showed that POEM was superior to LHM for type III achalasia with significantly better clinical response ( $98 \%$ vs $80.8 \%$ ), shorter procedure time (102 vs $264 \mathrm{~min}$ ) despite longer myotomy (16 vs $8 \mathrm{~cm}$ ) [3]. 

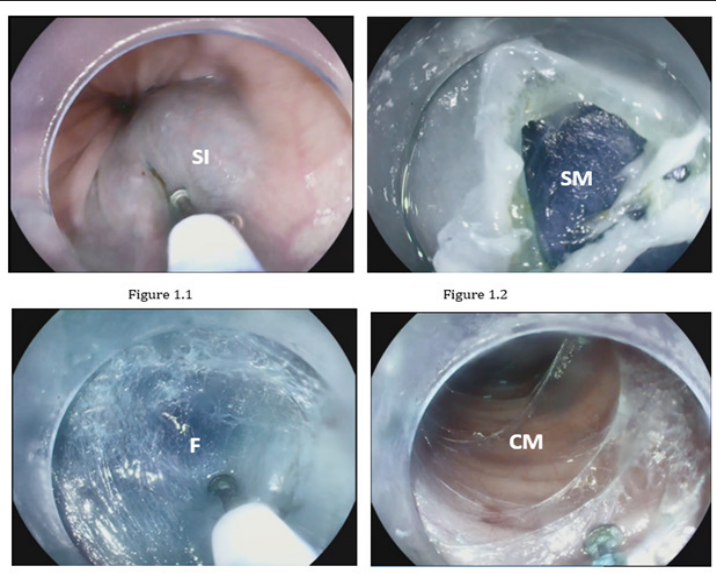

Figure 1.2

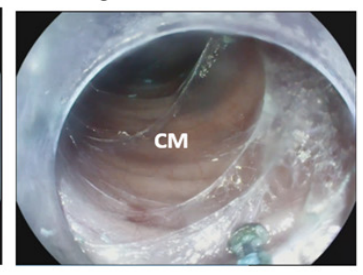

Figure 1.3

Figure 1.4
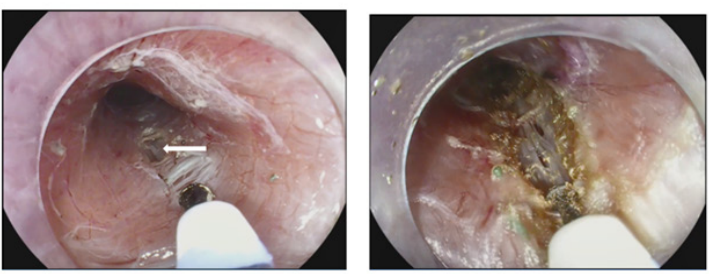

Figure 1.5

Figure 1.6|

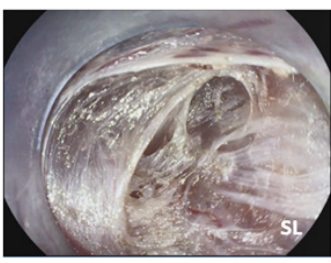

Figure 1.7

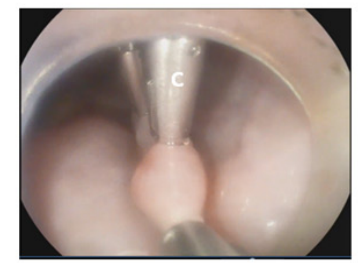

Figure 1.8

Figure 1: Per oral endoscopic myotomy (POEM)

1.1. Submucosal injection (SI) to create a cushion for the mucosal incision.

1.2. Longitudinal $2 \mathrm{~cm}$ incision exposing the submucosa (SM) using a Hybrid T-knife (mucosotomy).

1.3. Entrance into the submucosal with a cap-fitted upper endoscope exposing the blue stained submucosal fibers $(F)$.

1.4. Completed submucosal tunnel exposing the inner circular muscle (CM) of the muscularis propria (2-8 o'clock).

1.5. Initial incision of the circular muscle exposing the thin outer longitudinal muscle bundles (arrow).

1.6. Selective extension of the circular muscle layer.

1.7. Complete myotomy (circular and longitudinal) of the muscularis propria, exposing the thin outermost serosal layer (SL).

1.8. Closure of the mucosotomy with clips (C).

Submucosal tunneling may be performed at anterior 2 o'clock position or posterior 5 o'clock position. A meta-analysis of four trials enrolling 448 patients found posterior POEM to associated with fewer adverse events including lower risk of mucosotomy and shorter incision closure time [4] while another trial of 63 patients found no difference in short-term treatment efficacy, manometry outcomes, and adverse events [5]. Another difference in approach is the selective myotomy of inner circular bundles compared to full thickness myotomy. A study of 234 patients found comparable short term clinical outcomes but long term outcomes are awaited [6]. However, we prefer selective circular myotomy compared to full thickness myotomy to avoid entering the pleural space and the consequent increase in morbidity.

Clinical success is defined as a post-intervention Eckardt score of 3 or lower or a reduction of LES by $50 \%$ or more. In a metaanalysis of 7000 patients, POEM was more effective than LHM in improving dysphagia at 12-, 24-, 30- and 60-month intervals although POEM had higher incidence of reflux [1]. Newer suggested anti-reflux measures include combining POEM with transoral incisional fundoplication with one small study showing improved symptoms of esophagitis and lower need for long term proton pump inhibitor use [7].

\section{Spastic Esophageal Disorders (SED)}

This group comprises of diffuse esophageal spasm, nutcracker esophagus, hypercontractile (jackhammer) esophagus and spastic achalasia (Type III). Spastic achalasia and diffuse esophageal spasm have loss of neural inhibition, while jackhammer esophagus is believed to be caused by hypercontractility secondary to cholinergic pathway activation [8]. Medical management is reported to have failure rate of upto $74 \%$ in one study [9]. Access to the esophagus surgically may need access to the thorax and abdominal compartments. In LHM, access to the upper esophageal body may be challenging or limited. On the other hand, endoscopy has easy access to the entire esophageal body; therefore, allowing for an extended myotomy with precision. POEM, which was being 
performed for spastic achalasia, is now being indicated for spastic esophageal disorders. Myotomy is performed in areas of spastic esophageal contractions in the body of the esophagus as well as the lower esophageal sphincter. One meta-analysis showed POEM having a cumulative clinical success rate of $87 \%$ with a follow-up ranging from 3 months to 3 years, and also appeared to be effective in 6 patients who had symptom relapse after a prior Heller myotomy. Standardization of techniques of POEM for SEDs including the length of myotomy, approach (anterior, posterior, or both) may help to improve outcomes [10].

\section{Esophagogastric junction outflow obstruction (EGJ00)}

EGJOO is a new entity with little known about its etiology. The Chicago classification defined EGJOO as patients having an elevated median integrated relaxation pressure (IRP) on high resolution manometry with intact or weak peristalsis such that criteria for achalasia are not met [11]. The first step is to identify patients with secondary EGJOO due to mechanical obstruction with the help of barium esophagogram, upper endoscopy with biopsies, endoscopic ultrasound and/or CT chest, and treat them appropriately. The remaining patients fall into the idiopathic group, for whom a timed barium esophagram or EndoFLIP (endoluminal functional lumen imaging probe) is done to see if a true obstruction exists. If these tests confirm obstruction and the patient presents with dysphagia as the major complaint, then this group of patients is treated as a variant of achalasia with botulinum toxin, pneumatic dilation, or surgical myotomy. Other patients with normal values are managed medically with reassurance, calcium channel blockers, proton pump inhibitors and/or with simple esophageal dilation [12]. Richter et al reported good durable five-year symptom improvement with pneumatic dilation as first line treatment in 33 patients [12]. POEM has shown promise, but more data is needed at this time.

\section{Zenker Diverticulum}

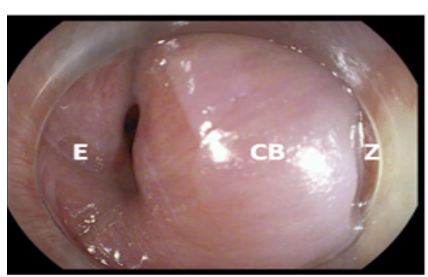

Figure 2.1

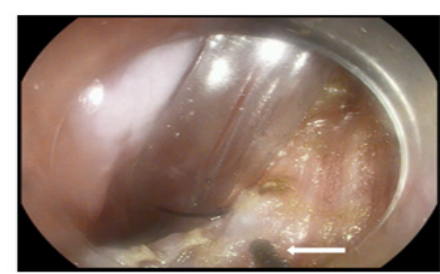

Figure 2.2

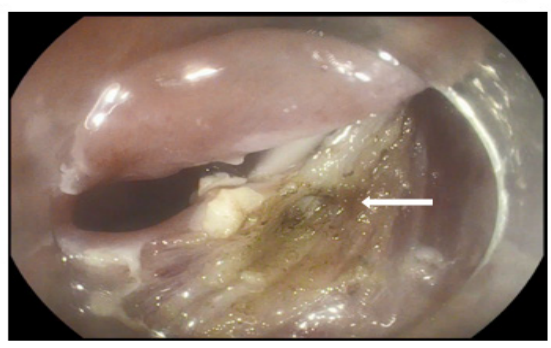

Figure 2.3

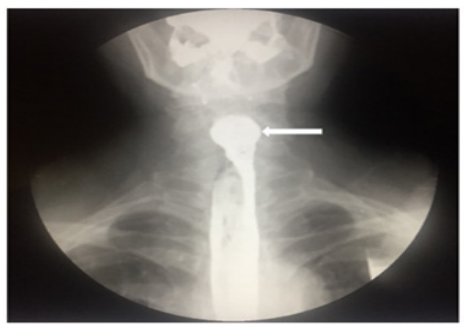

Figure 2.4

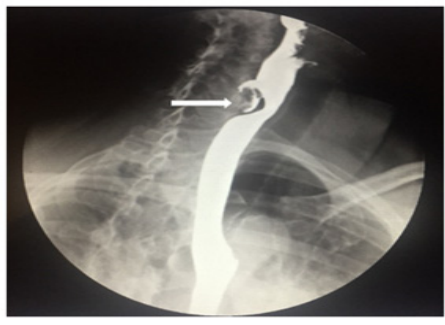

Figure 2.5

Figure 2: Zenker Diverticulum

2.1. Cricopharyngeal bar (CB) separating Zenker Diverticulum (Z) on the right and the esophageal lumen (E) on the left.

2.2. Myotomy using electrosurgical knife cutting individual fibers of the $C B$.

2.3. Completed myotomy of $\mathrm{CB}$.

2.4. Pre-procedure barium swallow demonstrating a $3 \mathrm{~cm}$ Zenker's Diverticulum.

2.5. Post myotomy barium swallow showing small residual cavity.

Zenker Diverticulum has been traditionally treated by open or rigid endoscopic stapling techniques. Endoscopic technique involves coagulation/incision of septum separating the diverticulum from the esophagus. Fibres of the cricopharyngeal muscle are incised with needle knives or argon plasma coagulation to prevent accumulation of food in the pouch (Figure 2). Comparing different modalities of therapy, success rates appear comparable (surgery: 80-100\%, rigid endoscopy: 90-100\%, flexible endoscopy: $43-100 \%$ ) but symptomatic recurrence can be as high as $19 \%$ for surgery, $12.8 \%$ for rigid endoscopy and $20 \%$ for flexible endoscopy [13]. 
Most of the techniques above involve incising of both the mucosa and muscle under limited visualization with little cue to whether the myotomy is complete. To overcome this, a modified technique called Z-POEM is being used to minimize the risk of mediastinitis or perforation. Mucosal incision is made on the apex of the septum followed by submucosal tunneling on both sides of the septum (esophageal and diverticular side). This allows for precise control of complete myotomy under better visualization. The short tunnel is then closed with mucosal clips [14].

\section{Per oral Pyloromyotomy}

Gastroparesis is a motility disorder of delayed gastric emptying often not responding to conventional medical treatments. Various interventions have been offered and these include gastric electric stimulation (GES), pyloric surgeries (PS) including pyloromyotomy and pyloroplasty, and gastrectomy. Pyloromyotomy has gained popularity due to the endoscopic approach. Called gastric POEM (G-POEM), the technique is similar to POEM but is performed in the gastric pylorus rather than the lower esophageal sphincter.
Pyloromyotomy is done on the greater curvature or the anterior gastric wall. A mucosal incision is done typically $5 \mathrm{~cm}$ proximal to the pylorus (Figure 3). The submucosal tunnel is extended to the pylorus and a myotomy is performed to completely incise the pylorus. In addition, the myotomy is extended for $2 \mathrm{~cm}$ along the antrum and closure can be done by clips or sutures. A study of 132 patients showed significantly improved Gastroparesis Cardinal Symptom Index (GCSI) scores after undergoing either GS, PS or combined GES+PS for gastroparesis refractory to medical treatment. On the other hand, GCSI nausea and vomiting subscale scores showed significant improvement with GES and combined GES+PS but not PS alone (Pre and post-operative scores. Nausea: GES $4.4 \pm 0.1$ to $3.3 \pm 0.3$; PS $3.5 \pm 0.4$ to $2.7 \pm 0.4$, GES+PS $4.1 \pm 0.3$ to $2.6 \pm 0.2$; Vomiting: GES $3.3 \pm 0.2$ to $2.3 \pm 0.3$, PS: $1.8 \pm 0.5$ to $1.3 \pm 0.4$, GES+PS $3.5 \pm 0.4$ to $1.8 \pm 0.5$ ) However, authors of this study also suggested a selection bias due to patients with nausea/ vomiting predominant symptoms being offered GES or GES+PS over PS [15].

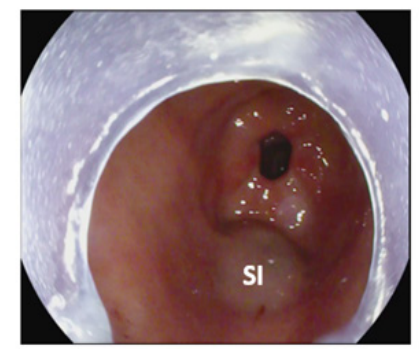

Figure 3.1

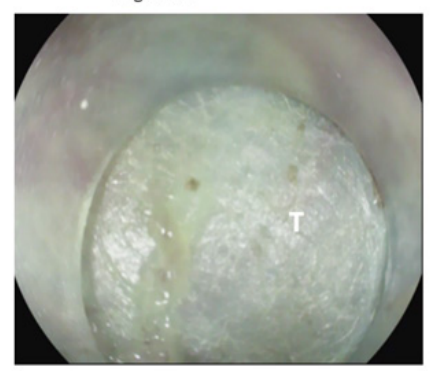

Figure 3.3

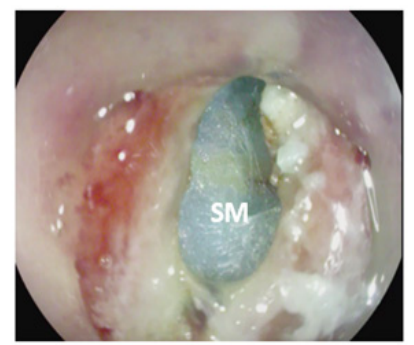

Figure 3.2

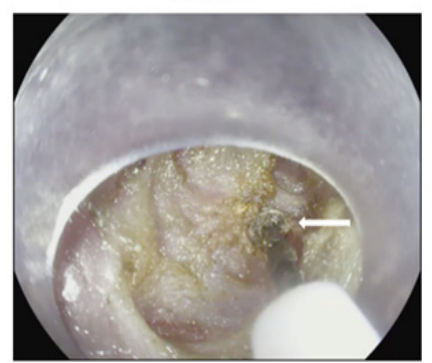

Figure 3.4

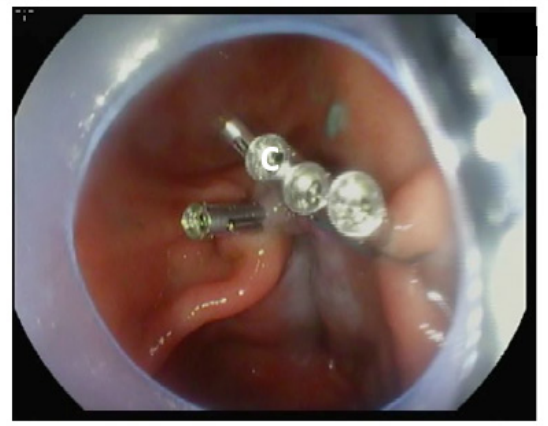

Figure 3.5

Figure 3: Gastric per oral endoscopic myotomy (G-POEM)

3.1 Gastric antrum: prepyloric submucosal injection (SI).

3.2 Completed mucosotomy exposing submucosa (SM).

3.3 Progression of submucosal tunnel $(\mathrm{T})$.

3.4 Dissection of pylorus with hybrid T-knife (arrow).

3.5 Closure of mucosotomy with clips $(\mathrm{C})$ completes pyloromyotomy. 


\section{References}

1. Ahmed Y, MO Othman (2019) Peroral endoscopic myotomy (POEM) for achalasia. Journal of Thoracic Disease S1618-S1628.

2. Andolfi C, PM Fisichella (2019) Meta-analysis of clinical outcome after treatment for achalasia based on manometric subtypes. Br J Surg 106(4): p. 332-341.

3. Kumbhari V, Tieu AH, Onimaru M, El Zein MH, Teitelbaum EN, et al (2015) Peroral endoscopic myotomy (POEM) vs laparoscopic Heller myotomy (LHM) for the treatment of Type III achalasia in 75 patients: a multicenter comparative study. Endosc Int Open 3(3): E195-E201.

4. Rodriguez de Santiago E, Mohammed N, Manolakis A, Shimamura Y, Onimaru M, et al. (2019) Anterior versus posterior myotomy during poem for the treatment of achalasia: systematic review and metaanalysis of randomized clinical trials. J Gastrointestin Liver Dis 28(1): 107-115.

5. Tan Y, Lv L, Wang X, Zhu H, Chu Y, et al. (2018) Efficacy of anterior versus posterior per-oral endoscopic\&\#xa0;myotomy for treating achalasia: a randomized, prospective study. Gastrointestinal Endoscopy 88(1): 4654.

6. Li QL, Chen WF, Zhou PH, Yao LQ, Xu MD, et al. (2013) Peroral Endoscopic Myotomy for the Treatment of Achalasia: A Clinical Comparative Study of Endoscopic Full-Thickness and Circular Muscle Myotomy. Journal of the American College of Surgeons 217(3): p. 442-451.

7. Tyberg A, Choi A, Gaidhane M, Kahaleh M (2018) Transoral Incisionless fundoplication for reflux after peroral endoscopic myotomy: a crucial addition to our arsenal. Endosc Int Open 6(05): E549-E552.
8. Roman S, PJ Kahrilas (2013) Management of Spastic Disorders of the Esophagus. Gastroenterology Clinics of North America 42(1): 27-43.

9. Patti MG, Pellegrini CA, Arcerito M, Tong J, Mulvihill SJ, et al. (1995) Comparison of Medical and Minimally Invasive Surgical Therapy for Primary Esophageal Motility Disorders. Arch Surg 130(6): 609-616.

10. Khan MA, Kumbhari V, Ngamruengphong S, Ismail A, Chen YI, et al. (2017) Is POEM the Answer for Management of Spastic Esophageal Disorders? A Systematic Review and Meta-Analysis. Digestive Diseases and Sciences 62(1): 35-44.

11. Kahrilas PJ, Bredenoord AJ, Fox M, Gyawali CP, Roman S, et al. (2015) The Chicago Classification of esophageal motility disorders, v3.0. Neurogastroenterology \& Motility 27(2): 160-174.

12. Richter JE, SB Clayton (2019) Diagnosis and Management of Esophagogastric Junction Outflow Obstruction. Am J Gastroenterol 114(4): 544-547.

13. Ishaq S, Sultan H, Siau K, Kuwai T, Mulder CJ, et al. (2018) New and emerging techniques for endoscopic treatment of Zenker's diverticulum: State-of-the-art review. Digestive Endoscopy 30(4): 449-460.

14. Chang KJ (2019) Endoscopic foregut surgery and interventions: The future is now. The state-of-the-art and my personal journey. World journal of gastroenterology 25(1): 1-41.

15. Zoll B, Jehangir A, Edwards MA, Petrov R, Hughes W, et al. (2019) Surgical Treatment for Refractory Gastroparesis: Stimulator, Pyloric Surgery, or Both? Journal of Gastrointestinal Surgery. 